\title{
Optimization of Applied Detection Rate in the Simple Evolving Connectionist System Method for Classification of Images Containing Protein
}

\author{
Rahmad Syah ${ }^{1}$, Al-Khowarizmi ${ }^{2}$ \\ ${ }^{1}$ Informatics Department, Universitas Medan Area, Medan, Indonesia \\ ${ }^{2}$ Information Teknlogi Department, Universitas Muhammadiyah Sumatera Utara, Medan, Indonesia
}

\begin{tabular}{l} 
ARTICLE INFO \\
\hline Article history: \\
Received April 03, 2021 \\
Revised April 18, 2021 \\
Accepted April 24, 2021 \\
\hline
\end{tabular}

\section{Keywords:}

Optimization; MAPE;

Detection rate;

Simple Evolving Connectionist;

Classification;

\begin{abstract}
Digital image processing, in general to makes images that appear converted to a function of light intensity represented in a two-dimensional plane. The function is a value that will be processed for classification so that the computer is able to recognize the image. Besides, classification requires training and testing to produce a small error value and optimal algorithm. The problem of optimization is closely related to the principles and findings of science. Getting the smallest error value by calculating using Mean Absolute Percent Error (MAPE) for that MAPE calculation is done by using the Detection Rate formula to generalize knowledge in order to find the optimal model. Thus, the application of Artificial Neural Network (ANN) is very suitable for optimizing classification using the Simple Evolving Connectionist System Method $(\mathrm{SECoS})$, and as a result, the classification of images containing protein with test data is that the eggs work with optimal proof of achieving MAPE without modification of $0.1947 \%$ and MAPE which has been modified with the formula detection rate of $0.05554633 \%$.
\end{abstract}

This work is licensed under a Creative Commons Attribution-Share Alike 4.0

\section{Rahmad Syah,}

Department of Informatics, Universitas Medan Area, Jln. Kolam, No.1, Medan, Indonesia.

Email: bayurahmadsyah45@gmail.com

\section{INTRODUCTION}

Digital image processing is included in the field of science that studies how an image is formed, processed, and analyzed to produce information that can be understood by humans [1][2]. Basically, digital image processing produces functions of light intensity that are represented in two dimensions [3]. Each image from the real world has various characteristics, which also consist of various knowledge [4]. For this reason, images need to be classified in order to be recognized and understood by computers quickly [5]. Meanwhile, many methods in digital image processing to explain data from an image by applying computer vision to explain data both manufacturing, medical, agriculture, and others that will be studied by the computer as computer knowledge [6]. One example carried out by reference [7] is to detect pests from tea leaves which are a good requirement of everyday human protein.

Protein is needed by humans, but in obtaining the protein, one must have knowledge of whether the protein is feasible or not suitable for consumption [8]. The feasibility of protein consumption needs to be reviewed and studied to get new knowledge contributions [9]. So that the use of computer science classifies people, so they do not hesitate in consuming protein by importing knowledge into the classification method.

Interest in this paper will be tested one of the protein data consumed daily with egg data. Eggs, in this case, chicken eggs are one source of animal protein that is very easy to obtain, and chicken eggs have nutritional value in them [10]. Reference [10] also examines digital image processing of chicken eggs using K-Nearest Neighbors to detect the quality of chicken eggs and has the results of getting the value $\mathrm{H}=7$ and accuracy of $86 \%$. At the same time, reference [11] in his paper conducted a study identifying chicken egg images with the Region of Interest Segmentation method and has an accuracy of $85 \%$ and an error of $15 \%$ in its application 
using a camera. In addition, reference [12] conducted a study to detect embryos in chicken egg images by using the Near-Infrared Hyperspectral Imaging method. The results were obtained when the first three bands with the maximum response of the spectral transmission characteristics were used, which showed the potential in applying imaging techniques. Hyperspectral to develop a real-time system for detecting fertility and early development of chicken egg embryos.

The automatic detection process requires a classification process [13]. Where is the process of one of the techniques in data mining that is used to find new models for certain purposes by utilizing data [14]. As reference [15] conducted a text classification using modified multi-class association rules, the proposed method was tested on a text classification problem, and the result shows that it performed better than the existing method in terms of classification accuracy and number of generated rules. Meanwhile reference [16] compares the Support Vector Machine (SVM) and the Multi-Layer Perceptron (MLP) Neural Networks for emotion classification, using prosodic and voice quality features extracted from the Berlin Emotional Database, is reported. Classification is very helpful in the process of learning on computers in computer knowledge working automatically [17][18].

Meanwhile, the big data process is mostly created due to the classification process by forecasting, prediction, association, and classification [19]. Reference [19] performs big data forecasting using the nearest neighbor method that focuses on when forecasting big data. Meanwhile, reference [20] also conducted big data forecasting to predict skin cancer using the evolving multilayer perceptron method. Evolving multilayer perceptron is the development of the multilayer perceptron method, which in some papers is called the Simple Evolving Connectionist System (SECoS) method [21]. SECoS consists of an input layer, hidden layer, and output layer where the development conducts training to multiply the hidden layer and output layer [22]. The process of success or failure at SECoS is measured using MAPE [23]. MAPE is the average of the overall percentage of error (difference) between the actual data with forecasting data that is measured and matched with time-series data and shown in percentage [24]. Meanwhile, to get the difference value based on the original value and forecast value can be calculated with the Detection rate formula [25]. So, the expectation of this paper is to get knowledge contribution by combining MAPE and detection rate in the process of classifying images containing protein as test data, namely egg images using the SECoS Method model to be optimal [26].

\section{MATERIAL AND METHOD}

\subsection{Image Histogram}

A histogram is a graph or diagram that shows the frequency of occurrence of each gradation in color [27]. Because it is a graph or diagram, the histogram displayed will have a horizontal axis and a vertical axis. The horizontal axis is the value of the color level, while the vertical, raise the frequency or number of pixels. From the image, a histogram will be used to get the characteristics or properties of statistical images. For more details, Fig. 1 is a histogram.

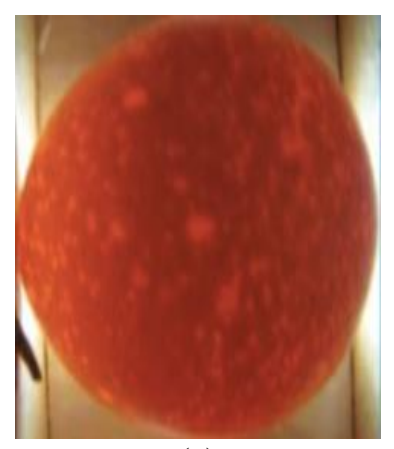

(a)

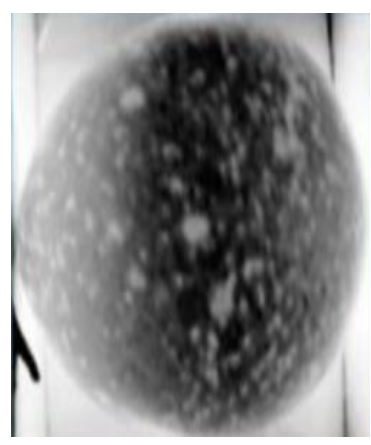

(b)

Fig. 1. Egg Image Histogram (a) original Image, (b) histogram Image

On Fig. 1 is an image of an egg which is done with a histogram. where the dataset in this paper uses egg images which will be outlined in the SECoS method. As for the training data and testing data, all of them are carried out by the histogram process. Histogram alignment is the act of changing the values of the intensity of the image so that the distribution is uniform. How to obtain it by changing the gray degree of a pixel $(r)$ with the new gray degree $(s)$ with a transformation function $T$, in this case, $s=T(r)$. The purpose of histogram leveling is to obtain an even distribution of the histogram so that each degree of gray has a relatively equal number of pixels. 


\subsection{General Architecture}

The system architecture is shown in Fig. 2. Explanation of steps in Fig. 2 as follows [28].

1. Do feature extraction on chicken egg image.

2. Learning rate, which, at this stage, will be weighed against adaptive learning, and sensitivity threshold are representations of input parameter values.

3. Before starting the data processing, the data to be trained is normalized to include a range of 0 to1, as shown in (1).

$$
y=\frac{x-x_{\min }}{x_{\max }-x_{\min }}
$$

Where $x$ is Feature extraction of egg images value, $y$ is Normalization value, $x_{\min }$ is Min. Value Feature extraction of egg images and $x_{\max }$ is Max. Values Feature extraction of egg images value.

4. Create the first layer and its node (r1), and set the Wi input weight to 1 based on the input vector I and the output weight $\mathrm{W} 1$ based on the Od vector output.

5. Calculate the activation value for each node that exists using the normalized Hamming distance formula, then proceed with the evolving multilayer perceptron calculation.

a. Look for nodes with quality decision acceleration as the parameter for sensitivity threshold in the evolving layer.

b. If a node's activation value is greater than the sensitivity threshold parameter, the data should be propagated to the output layer.

c. Obtain the output layer error value.

6. Perform steps 1 through 5 in data training.

7. Identify the node with the highest activation value and pass the data through it to the output layer.

8. Using (2), check the magnitude of the mistake with MAPE (Mean Absolute Percentage Error),

$$
M A P E=\sum_{t=1}^{n} \frac{\left|\frac{a-b}{a}\right|}{n} 100 \%
$$

Where $a$ is actually the data, $b$ is resulting in the data, and $n$ is a lot`s of data.

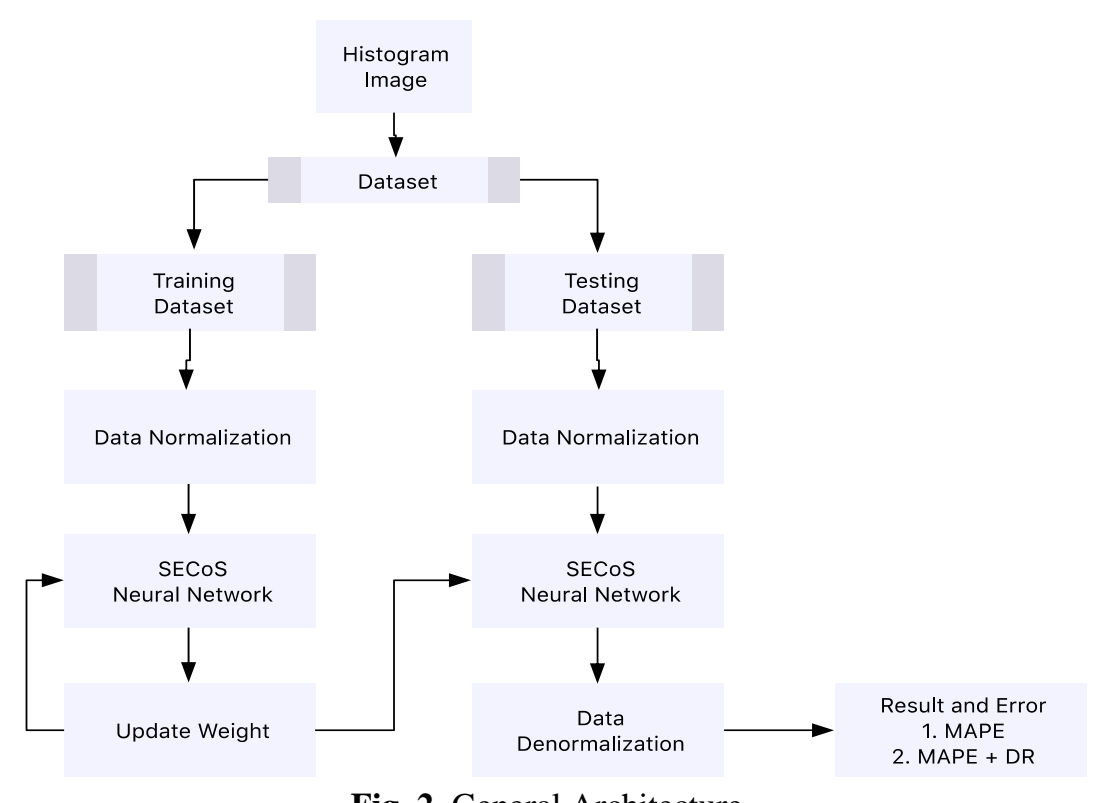

Fig. 2. General Architecture

An optimization process is done by calculating the difference in value with the rate of detection in (3).

$$
D R=\frac{T V}{T V+F V}
$$

where $T V$ is true value, $F V$ is false value.

The final result is calculated by Mean Absolute Percent Error (MAPE) combined with Detection Rate. The equation (4) of MAPE is as 


$$
M A P E=\frac{\sum_{t=1}^{n}|D R|}{n} \times 100 \%
$$

where $n$ is the amount of data.

\section{RESULT AND DISCUSSION}

Image histogram alignment changes the distribution of the degree values of grayscale in an image so that it is uniform. After getting the value of a histogram level, then analyzed the image with first-order statistical analysis. First-order statistical feature extraction has analytical value as a benchmark starting from the mean, standard deviation, bias, and kurtosis. The four values will be used as a parameter or attribute to determine the class of chicken eggs to be studied. Of all the processes that have been carried out so that all the data desired for research is collected, especially the sample data, the data will be further divided to be processed by the evolving multilayer perceptron method and the exchange of adaptive learning values, error threshold and sensitivity threshold will be exchanged later. The dataset was taken with 30 data consisting of ordinary chicken eggs and omega chicken eggs. The dataset was obtained from previous studies by reference [10]. The dataset is then normalized according to (1). The results are in Table 1.

Table 1. Normalization of the Egg Imagery Histogram

\begin{tabular}{cccccc}
\hline Object & \multicolumn{4}{c}{ Input } & y \\
\cline { 2 - 5 } Object 1 & Mean & Deviation & Skewness & Kurtosis & 0 \\
Object 2 & 0.68834338 & 0.97863016 & 0.01870345 & 0 & 0 \\
Object 3 & 0.752085617 & 0.78927675 & 0.20262176 & 0.08926514 & 0 \\
Object 4 & 0.66467604 & 0.79915353 & 0.17529249 & 0.07514038 & 0 \\
Object 5 & 0.87613481 & 0.6963247 & 0.28536891 & 0.14126026 & 0 \\
Object 6 & 0.31009127 & 0.66874499 & 0.32849801 & 0.12556555 & 0 \\
Object 7 & 0.95731615 & 0.70022522 & 0.25302207 & 0.1692615 & 0 \\
Object 8 & 0.18050755 & 0.77677135 & 0.23510831 & 0.108381271 & 0 \\
Object 9 & 0.77472448 & 0.8943059 & 0.08715725 & 0.03029879 & 0 \\
Object 10 & 0.94101413 & 0.90120321 & 0.06756691 & 0.02361829 & 0 \\
Object 11 & 0.44374974 & 0.97601279 & 0.03877368 & 0.00828502 & 0 \\
Object 12 & 0.56334111 & 1 & 0 & 0.00536043 & 0 \\
Object 13 & 1 & 0.8972326 & 0.06813792 & 0.02200591 & 0 \\
Object 14 & 0.61862623 & 0.97877682 & 0.02527001 & 0.00296545 & 0 \\
Object 15 & 0.78109147 & 0.89350685 & 0.08720584 & 0.03033494 & 0 \\
Object 16 & 0 & 0 & 0.99357316 & & 1 \\
Object 17 & 0.88803935 & 0.08766281 & 0.92214892 & 0.73978241 & 0 \\
Object 18 & 0.66616599 & 0.28951815 & 0.63811641 & 0.50225434 & 0 \\
Object 19 & 0.79459153 & 0.42989433 & 0.53100436 & 0.32853445 & 0 \\
Object 20 & 0.59944685 & 0.0443114 & 1 & 0.83542633 & 1 \\
Object 21 & 0.3896409 & 0.54160794 & 0.44421766 & 0.25494831 & 1 \\
Object 22 & 0.82825422 & 0.69505408 & 0.26769812 & 0.13007108 & 1 \\
Object 23 & 0.96803839 & 0.70013264 & 0.25043433 & 0.11941779 & 1 \\
Object 24 & 0.38894418 & 0.7863202 & 0.21342834 & 0.09547263 & 1 \\
Object 25 & 0.66334292 & 0.68680916 & 0.28683894 & 0.14119583 & 1 \\
Object 26 & 0.88803935 & 0.08766281 & 0.92214892 & 0.73978241 & 0 \\
Object 27 & 0.66616599 & 0.28951815 & 0.63811641 & 0.50225434 & 0 \\
Object 28 & 0.79459153 & 0.42989433 & 0.53100436 & 0.32853445 & 0 \\
Object 29 & 0.59944685 & 0.0443114 & 1 & 0.83542633 & 1 \\
Object 30 & 0.3896409 & 0.54160794 & 0.44421766 & 0.25494831 & 1 \\
\hline
\end{tabular}

Table 1 is the data of chicken eggs that have been photographed and then do histogram levelling to produce mean, standard deviation, skewness, kurtosis, and entropy. After getting the parameter values normalized data with a range of values from 0 to 1 . After getting the normalized values, training data is done with the artificial conditions network, as shown in Fig. 3.

Fig. 3 is a network of SECoS where the process is available in 3 layers, namely the input layer, hidden layer and output layer. The input layer will include variables, namely the result of the histogram data in the form of mean, deviation, skewness, and kurtosis. Then the weight and input values are calculated which are regulated by the activation value so that they enter the hidden layer, where in the hidden layer there are differences in the SECoS network with other artificial neural networks such as adding nodes that have expanded or envolved. As for the final result in the form of an output layer which will also increase the number of nodes based on the activation value.

Optimization of Applied Detection Rate in the Simple Evolving Connectionist System Method for Classification of Images Containing Protein (Rahmad Syah) 


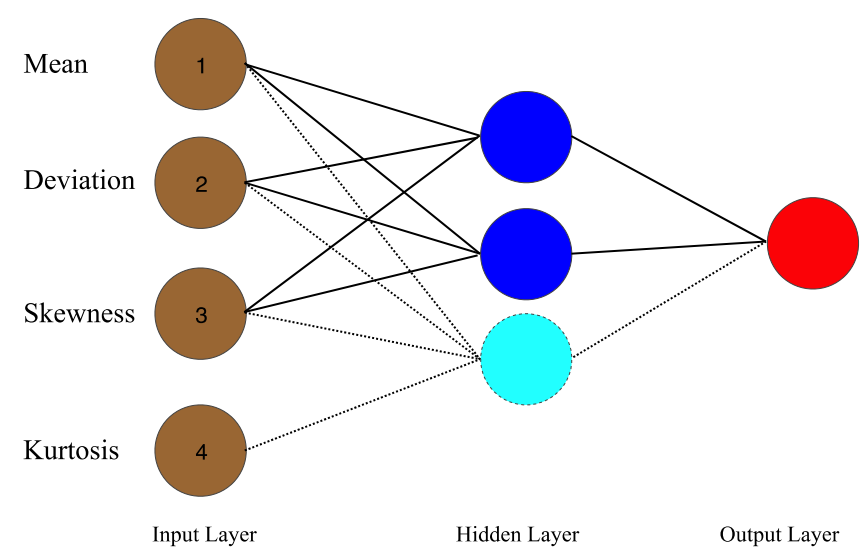

Fig. 3. Artificial Neural Network

The four normalized parameter data are trained using evolving multilayer perceptron. In training, applied evolving multilayer perceptron, determine the parameter value. The learning rate will be used as a parameter value $1=0.9$, the rate of learning $2=0.1$, Threshold for sensitivity $=0.9$, threshold for Error $=0.1$. Then input the layer up to the output layer with the weight value, $\mathrm{W}_{1}, \mathrm{~W}_{2} \mathrm{~W}_{3}, \mathrm{~W}_{4}$, and $\mathrm{W}_{5}$. As an initial weight, the first pair of vectors input-output is used $\mathrm{W}_{1}=0.68834338, \mathrm{~W}_{2}=0,97863016, \mathrm{~W}_{3}=0,01870345, \mathrm{~W}_{4}=0$ and $\mathrm{W}_{5}=$ 0 , where if W5 is zero then eggs without omega-3 if w5 1 then eggs have omega-3. After doing training data, then testing the data where the testing data is also done with the same data to get the MAPE value. Table 2 shows the results of this method.

Table 2. Vector data and Error

\begin{tabular}{ccccc}
\hline Input & Classification target & Error & Detection Rate & Object \\
\hline $\mathbf{0 . 4 2 1 4 1 9 2 4 8}$ & 0.39375346 & 0,02766579 & 0.51696928 & Object 1 \\
$\mathbf{0 . 3 9 3 7 5 3 4 5 8}$ & 0.45041801 & -0.0566645 & 0.46643777 & Object 2 \\
$\mathbf{0 . 4 5 0 4 1 8 0 0 7}$ & 0.44470294 & 0.00571507 & 0.50319234 & Object 3 \\
$\mathbf{0 . 4 4 4 7 0 2 9 3 7}$ & 0.48986357 & -0.0451606 & 0.47583873 & Object 4 \\
$\mathbf{0 . 4 8 9 8 6 3 5 6 6}$ & 0.36914894 & 0.12071462 & 0.5702636 & Object 5 \\
$\mathbf{0 . 3 6 9 1 4 8 9 4 2}$ & 0.50781904 & -0.1386701 & 0.42093776 & Object 6 \\
$\mathbf{0 . 5 0 7 8 1 9 0 4 1}$ & 0.32519206 & 0.18262698 & 0.60961857 & Object 7 \\
$\mathbf{0 . 3 2 5 1 9 2 0 6 3}$ & 0.44662161 & -0.1214295 & 0.42133494 & Object 8 \\
$\mathbf{0 . 4 4 6 6 2 1 6 0 5}$ & 0.48335064 & -0.036729 & 0.48025262 & Object 9 \\
$\mathbf{0 . 4 8 3 3 5 0 6 3 5}$ & 0.36670531 & 0.11664533 & 0.56861038 & Object 10 \\
$\mathbf{0 . 3 6 6 7 0 5 3 0 6}$ & 0.39217539 & -0.0254701 & 0.48321865 & Object 11 \\
$\mathbf{0 . 3 9 2 1 7 5 3 8 7}$ & 0.49684411 & -0.1046687 & 0.44113249 & Object 12 \\
$\mathbf{0 . 4 9 6 8 4 4 1 0 6}$ & 0.40640963 & 0.09043448 & 0.5500604 & Object 13 \\
$\mathbf{0 . 4 0 6 4 0 9 6 2 6}$ & 0.44803477 & -0.0416251 & 0.47564198 & Object 14 \\
$\mathbf{0 . 4 4 8 0 3 4 7 7 4}$ & 0.49839329 & -0.0503585 & 0.47339549 & Object 15 \\
$\mathbf{0 . 4 9 8 3 9 3 2 8 9}$ & 0.65940837 & -0.1610151 & 0.43046517 & Object 16 \\
$\mathbf{0 . 6 5 9 4 0 8 3 7 3}$ & 0.52401372 & 0.13539465 & 0.55720472 & Object 17 \\
$\mathbf{0 . 5 2 4 0 1 3 7 2 4}$ & 0.52100617 & 0.00300756 & 0.50143899 & Object 18 \\
$\mathbf{0 . 5 2 1 0 0 6 1 6 8}$ & 0.61979614 & -0.09879 & 0.45670154 & Object 19 \\
$\mathbf{0 . 6 1 9 7 9 6 1 4 3}$ & 0.4076037 & 0.21219244 & 0.60326673 & Object 20 \\
$\mathbf{0 . 4 0 7 6 0 3 7 0 2}$ & 0.48026938 & -0.0726657 & 0.45907879 & Object 21 \\
$\mathbf{0 . 4 8 0 2 6 9 3 7 6}$ & 0.50950579 & -0.0292364 & 0.48523078 & Object 22 \\
$\mathbf{0 . 5 0 9 5 0 5 7 8 6}$ & 0.37104134 & 0.13846445 & 0.5786241 & Object 23 \\
$\mathbf{0 . 3 7 1 0 4 1 3 3 8}$ & 0.44454671 & -0.0735054 & 0.45493719 & Object 24 \\
$\mathbf{0 . 4 4 4 5 4 6 7 1 2}$ & 0.65940837 & -0.2148617 & 0.40268551 & Object 25 \\
$\mathbf{0 . 6 5 9 4 0 8 3 7 3}$ & 0.52401372 & 0.13539465 & 0.55720472 & Object 26 \\
$\mathbf{0 . 5 2 4 0 1 3 7 2 4}$ & 0.52100617 & 0.00300756 & 0.50143899 & Object 27 \\
$\mathbf{0 . 5 2 1 0 0 6 1 6 8}$ & 0.61979614 & -0.09879 & 0.45670154 & Object 28 \\
$\mathbf{0 . 6 1 9 7 9 6 1 4 3}$ & 0.4076037 & 0.21219244 & 0.60326673 & Object 29 \\
$\mathbf{0 . 4 0 7 6 0 3 7 0 2}$ & 0.42141925 & -0.0138155 & 0.49166757 & Object 30 \\
\hline
\end{tabular}

After getting the value in classifying as well as the error value, the last step calculates MAPE with (2). So, the MAPE value obtained is $0.1947 \%$. Besides that, to get knowledge contribution based on process classification, optimization of the MAPE formula is combined with the detection rate formula, and the MAPE results using the detection rate formula are calculated with (3) and (4) of $0.05554633 \%$. So that the MAPE value that uses the detection rate is a very small value so that in this paper and the contribution of knowledge

Optimization of Applied Detection Rate in the Simple Evolving Connectionist System Method for Classification of Images Containing Protein (Rahmad Syah) 
to the Simple Evolving Connectionist System $(\mathrm{SECoS})$ method get a value of accuracy that is large enough to classify the image that contains protein. Thus, the detection rate is able to provide optimal values for the MAPE measuring instrument so that further research needs to do optimization in addition to using detection rates in other classification methods.

\section{CONCLUSION}

In summary, this paper classifies using the SECoS Method on images containing protein with test data being eggs. Where the classification works optimally, this is proven by optimizing the MAPE calculations combined with the detection rate. The results of the classification process with SECoS get MAPE value without using a direction rate of $0.1947 \%$ and MAPE value using a direction rate of $0.05554633 \%$. MAPE that uses the detection rate includes a very small error value so that the measurement of accuracy in the classification is quite optimal for the SECoS method.

\section{REFERENCES}

[1] A. V. Iatsyshyn et al., "Formation of the scientist image in modern conditions of digital society transformation," In Journal of Physics: Conference Series, vol. 1840, no. 1, pp. 012039, 2021, IOP Publishing. https://doi.org/10.1088/1742-6596/1840/1/012039

[2] R. Sa'ari, N. A. Rahman, Z. M. Yusof, S.K. Ngien, S.A. Kamaruddin, and M.A. Hezmi, "Application of digital image processing technique in monitoring LNAPL migration in double porosity soil column," Jurnal Teknologi, vol. 72, no. 3, pp. 23-29, 2015. https://doi.org/10.11113/jt.v72.4018

[3] A.M. Alawad, A. Rahman, F. Diyana, O.O. Khalifa, and N.A. Malek, "Fuzzy Logic based Edge Detection Method for Image Processing," International Journal of Electrical \& Computer Engineering, vol. 8, no. 3, pp. 2088-8708, 2018. https://doi.org/10.11591/ijece.v8i3.pp1863-1869

[4] A. Khmag, S. Ghoul, S.A.R. Al-Haddad, and N. Kamarudin, "Noise Level Estimation for Digital Images Using Local Statistics and Its Applications to Noise Removal," Telkomnika, vol. 16, no. 2, pp.915-924, 2018. https://doi.org/10.12928/telkomnika.v16i2.9060

[5] M. Nixon and A. Aguado, "Feature extraction and image processing for computer vision," Academic Press, 2019. https://doi.org/10.1016/B978-0-12-814976-8.00003-8

[6] H.A. Wibawa and P.S. Sasongko, "Detection of Ship using Image Processing and Neural Network," Telkomnika, vol. 16, no. 1, pp.259-264, 2018. https://doi.org/10.12928/telkomnika.v16i1.7357

[7] A. R. Lubis, S. Prayudani, M. Lubis, and A. Al-Khowarizmi, "Decision Making in the Tea Leaves Diseases Detection Using Mamdani Fuzzy Inference Method, “Indonesian Journal of Electrical Engineering and Computer Science, vol. 12, no. 3, 2018. https://doi.org/10.11591/ijeecs.v12.i3.pp1273-1281

[8] M. Pojić, A. Mišan, and B. Tiwari, "Eco-innovative technologies for extraction of proteins for human consumption from renewable protein sources of plant origin," Trends in Food Science \& Technology, vol. 75, pp. 93-104, 2018. https://doi.org/10.1016/j.tifs.2018.03.010

[9] C. Hartmann and M. Siegrist, "Consumer perception and behaviour regarding sustainable protein consumption: A systematic review," Trends in Food Science \& Technology, vol. 61, pp. 11-25, 2017. https://doi.org/10.1016/j.tifs.2016.12.006

[10] R. Rahmadianto, E. Mulyanto, and T. Sutojo, "Implementasi Pengolahan Citra dan Klasifikasi K-Nearest Neighbor untuk Mendeteksi Kualitas Telur Ayam,” Jurnal VOI (Voice of Informatics), vol. 8, no. 1, 2019.

[11] A. Muzami, O.D. Nurhayati, and K.T. Martono, "Aplikasi Identifikasi Citra Telur Ayam Omega-3 Dengan Metode Segmentasi Region of Interest Berbasis Android," Jurnal Teknologi dan Sistem Komputer, vol. 4, no. 2, pp.380-388, 2016. https://doi.org/10.14710/jtsiskom.4.2.2016.380-388

[12] H. Jiang, S. C. Yoon, H. Zhuang, W. Wang, K.C. Lawrence, and Y. Yang, "Tenderness classification of fresh broiler breast fillets using visible and near-infrared hyperspectral imaging," Meat science, vol. 139, pp. 82-90, 2018. https://doi.org/10.1016/j.meatsci.2018.01.013

[13] M. S. A. M. Ali, A.H. Jahidin, M. N. Taib, and N. M. Tahir, "EEG sub-band spectral centroid frequency and amplitude ratio features: A comparative study in learning style classification," Jurnal Teknologi, vol. 78, no. 2, 2016. https://doi.org/10.11113/jt.v78.4100

[14] R. Syah, M. K. M Nasution, E. B Nababan, S. Efendi, "Sensitivity of shortest distance search in the ant colony algorithm with varying normalized distance formulas.," TELKOMNIKA, vol. 19, no. 4, 2021. http://dx.doi.org/10.12928/telkomnika.v19i4.18872

[15] S. Prayudani, A. Hizriadi, Y. Y. Lase, Y. Fatmi, and A. Al-Khowarizmi, "Analysis Accuracy of Forecasting Measurement Technique on Random K-Nearest Neighbor (RKNN) Using MAPE and MSE,” In Journal of Physics: Conference Series, vol. 1361, no. 1, pp. 012089), 2019. IOP Publishing. https://doi.org/10.1088/17426596/1361/1/012089

[16] S. S. Kamaruddin, Y. Yusof, H. Husni, and M.H. Al Refai, "Text classification using modified multi class association rule," Jurnal Teknologi, vol. 78, no. 8, 2016. https://doi.org/10.11113/jt.v78.9553

[17] Al-Khowarizmi, R. Syah, M. K. M. Nasution, M. Elveny. Sensitivity of MAPE using Detection Rate for Big Data Forecasting Crude Palm Oil on k-Nearest Neighbor. Vol. 19, no. 4. International Journal of Electrical and Computer Engineering (IJECE), vol. 11, no. 3, 2021. https://doi.org/10.11591/ijece.v11i3.pp2696-2703 
[18] B. Bozkurt, I. Germanakis, and Y. Stylianou, "A study of time-frequency features for CNN-based automatic heart sound classification for pathology detection," Computers in biology and medicine, vol. 100, pp.132-143, 2018. https://doi.org/10.1016/j.compbiomed.2018.06.026

[19] N. L. Hairuddin, L. M. Yusuf, M. S. Othman, and H. A. Majid,” Improving Gender Classification with Feature Selection in Forensic Anthropology," Jurnal Teknologi, vol. 78, no. 12, 2016. https://doi.org/10.11113/jt.v78.10143

[20] A. Al-Khowarizmi and S. Suherman," Classification of skin cancer images by applying simple evolving connectionist system," IAES International Journal of Artificial Intelligence (IJ-AI), vol. 10, no. 2, 2021.

[21] A. R. Lubis, M. Lubis, A. Al-Khowarizmi and D. Listriani, "Big Data Forecasting Applied Nearest Neighbor Method," In 2019 International Conference on Sustainable Engineering and Creative Computing (ICSECC), pp. 116-120, 2019. https://doi.org/10.1109/ICSECC.2019.8907010

[22] R. Syah., M. Nasution, M. Elveny, and H. Arbie," Optimization Model for Customer Behavior with Mars and KYC System," Journal of Theoretical and Applied Information Technology, vol. 98, no. 13, 2020.

[23] N. K Kasabov, "Artificial neural networks. evolving connectionist systems," In Time-Space, Spiking Neural Networks and Brain-Inspired Artificial Intelligence, pp. 39-83, 2019, Springer, Berlin, Heidelberg. https://doi.org/10.1007/978-3-662-57715-8_2

[24] A. Al-Khowarizmi, O. S. Sitompul, S. Suherman, and E.B Nababan, "Measuring the Accuracy of Simple Evolving Connectionist System with Varying Distance Formulas," In Journal of Physics: Conference Series, vol. 930, no. 1, 2017. IOP Publishing. https://doi.org/10.1088/1742-6596/930/1/012004

[25] A. Al-Khowarizmi, I. R. Nasution, M. Lubis, and A.R. Lubis, "The effect of a SECoS in crude palm oil forecasting to improve business intelligence," Bulletin of Electrical Engineering and Informatics, vol. 9, no. 4, 2020. https://doi.org/10.11591/eei.v9i4.2388

[26] A. Al-Khowarizmi, "Modification of the SECoS Method uses the Distance Formula," Thesis Computer Science, Universitas Sumatera Utara, 2017.

[27] A. Y. Lam, Y. Li, D. L. Gregory, J. Prinz, J. O’Reilly, M. Manka, and R.N. Keswani, “Association between improved adenoma detection rate and interval colorectal cancer rates after a quality improvement program," Gastrointestinal Endoscopy, 2020. https://doi.org/10.1016/j.gie.2020.02.016

[28] A. R. Lubis, M. Lubis, and Al-Khowarizmi," Optimization of distance formula in K-Nearest Neighbor method," Bulletin of Electrical Engineering and Informatics, vol. 9, no. 1, pp. 326-338, 2020. https://doi.org/10.11591/eei.v9i1.1464

\section{BIOGRAPHY OF AUTHORS}

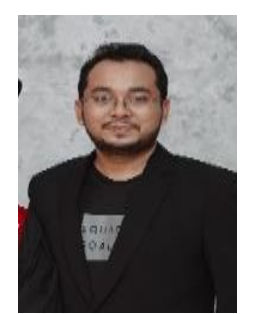

Rahmad Syah is a Lecturer from the University of Medan Area, Informatics Department Study Program. Completing a Doctoral Program (S3) at the University of Sumatra Utara (USU) in 2021. He obtained a Master's Degree (S2) in Informatics in 2013 from University of Sumatera Utara (USU), Medan. Undergraduate education (S1) at the Universitas Komputer Indonesia (UNIKOM) in 2011. Research focuses on Computing Modelling and Systems studies, Information Systems, Business Intelligence and Analytics (BIA), Artificial Intelligence, Data Science.

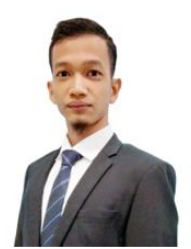

Al-Khowarizmi was born in Medan, Indonesia, in 1992. He is a lecturer at Universitas Muhammadiyah Sumatera Utara (UMSU), Medan, Indonesia, in the Information Technology Department, Faculty of Computer Science and Information Technology. Computer Science, Neural Networks, Artificial Intelligence, Big Data, Business Intelligence and Analytics, Machine Learning are among his core research interests. 